\title{
Dosing of thromboprophylaxis and mortality in critically ill COVID-19 patients
}

Sandra Jonmarker ${ }^{1,2}$, Jacob Hollenberg ${ }^{3}$, Martin Dahlberg ${ }^{1,4}$, Otto Stackelberg ${ }^{1,4,5}$, Jacob Litorell ${ }^{2}$, Åsa H. Everhov ${ }^{1,4}$, Hans Järnbert-Pettersson' ${ }^{1}$, Mårten Söderberg ${ }^{1,6}$, Jonathan Grip ${ }^{7,8}$, Anna Schand1 2,9, Mattias Günther ${ }^{1,2}$ and Maria Cronhjort ${ }^{1,2^{*}}$

\begin{abstract}
Background: A substantial proportion of critically ill COVID-19 patients develop thromboembolic complications, but it is unclear whether higher doses of thromboprophylaxis are associated with lower mortality rates. The purpose of the study was to evaluate the association between initial dosing strategy of thromboprophylaxis in critically ill COVID19 patients and the risk of death, thromboembolism, and bleeding.

Method: In this retrospective study, all critically ill COVID-19 patients admitted to two intensive care units in March and April 2020 were eligible. Patients were categorized into three groups according to initial daily dose of thromboprophylaxis: low (2500-4500 IU tinzaparin or 2500-5000 IU dalteparin), medium ( $>4500 \mathrm{IU}$ but $<175 \mathrm{IU} / \mathrm{kilogram,}$ $\mathrm{kg}$, of body weight tinzaparin or $>5000 \mathrm{IU}$ but $<200 \mathrm{IU} / \mathrm{kg}$ of body weight dalteparin), and high dose ( $\geq 175 \mathrm{IU} / \mathrm{kg}$ of body weight tinzaparin or $\geq 200 \mathrm{IJ} / \mathrm{kg}$ of body weight dalteparin). Thromboprophylaxis dosage was based on local standardized recommendations, not on degree of critical illness or risk of thrombosis. Cox proportional hazards regression was used to estimate hazard ratios with corresponding 95\% confidence intervals of death within 28 days from ICU admission. Multivariable models were adjusted for sex, age, body mass index, Simplified Acute Physiology Score III, invasive respiratory support, and initial dosing strategy of thromboprophylaxis.
\end{abstract}

Results: A total of 152 patients were included: 67 received low-, 48 medium-, and 37 high-dose thromboprophylaxis. Baseline characteristics did not differ between groups. For patients who received high-dose prophylaxis, mortality was lower (13.5\%) compared to those who received medium dose (25.0\%) or low dose (38.8\%), $p=0.02$. The hazard ratio of death was 0.33 (95\% confidence intervals $0.13-0.87$ ) among those who received high dose, and 0.88 (95\% confidence intervals $0.43-1.83$ ) among those who received medium dose, as compared to those who received low-dose thromboprophylaxis. There were fewer thromboembolic events in the high (2.7\%) vs medium (18.8\%) and low-dose thromboprophylaxis (17.9\%) groups, $p=0.04$.

Conclusions: Among critically ill COVID-19 patients with respiratory failure, high-dose thromboprophylaxis was associated with a lower risk of death and a lower cumulative incidence of thromboembolic events compared with lower doses.

Trial registration: Clinicaltrials.gov NCT04412304 June 2, 2020, retrospectively registered.

Keywords: Anticoagulation, COVID-19, Critical care, Low molecular weight heparin, SARS-CoV-2, Thromboembolism

*Correspondence: maria.cronhjort@sll.se

${ }^{1}$ Department of Clinical Science and Education, Södersjukhuset, Karolinska Institutet, Stockholm, Sweden

Full list of author information is available at the end of the article

\section{Background}

The inflammatory response to Coronavirus disease 19 (COVID-19) seems to trigger thrombotic activation in the venous and the arterial circulation [1,2]. Autopsy findings suggest that this coagulopathy occurs in otherwise in a credit line to the material. If material is not included in the article's Creative Commons licence and your intended use is not permitted by statutory regulation or exceeds the permitted use, you will need to obtain permission directly from the copyright holder. To view a copy of this licence, visit http://creativecommons.org/licenses/by/4.0/. The Creative Commons Public Domain Dedication waiver (http://creativecommons.org/publicdomain/zero/1.0/) applies to the data made available in this article, unless otherwise stated in a credit line to the data. 
micro- as well as macrovascular beds [3-5]. As many as $17-69 \%$ of COVID-19 patients in intensive care units (ICUs) suffer from thrombotic events and $13-35 \%$ are diagnosed with pulmonary embolism (PE) [6-9]. This is significantly more than in non-COVID-19 acute respiratory distress syndrome patients [8]. For COVID-19 patients, laboratory findings indicate a hypercoagulable state in combination of low grade disseminated intravascular coagulation and thrombotic angiopathy $[10,11]$ which differ from what is typically seen in sepsis [12]. In previous studies, high levels of Fibrin-D-dimer and $C$-reactive protein (CRP) have been associated with poor outcome in COVID-19 [8,11-14]. In an observational study of hospitalized COVID-19 patients, anticoagulation was associated with improved outcome among mechanically ventilated COVID-19 patients [15], but the optimal choice of dose is yet to be determined. The risk of bleeding with full-dose anticoagulants has been described in a small retrospective study where $21 \%$ had hemorrhagic events despite anti-factor $\mathrm{Xa}$ activity within the therapeutic range for all patients except one [16].

At Södersjukhuset, Stockholm, Sweden, the first critically ill COVID-19 patients admitted to the ICUs were treated with low-dose thromboprophylaxis. Within a few weeks, after preliminary reports suggesting that a high proportion of COVID-19 patients suffered from thromboembolic events, it was decided to increase the dose of thromboprophylaxis.

The objective of this study was to evaluate the association between dosing strategy of thromboprophylaxis in critically ill COVID-19 patients with respiratory failure and the risk of death, thromboembolism and bleeding.

\section{Methods}

\section{Trial overview and patients}

In this retrospective, observational cohort study, all critically ill COVID-19 patients (verified with polymerase chain reaction-positive Severe Acute Respiratory Syndrome Corona Virus 2, SARS-CoV-2) with respiratory failure, admitted to two ICUs in March and April, 2020, at Södersjukhuset, Stockholm, Sweden, were eligible for inclusion. The study was approved by the regional ethical review board in Uppsala, Sweden, (Dnr: 2020-01302, amendment 2020-02890), and informed consent was waived.

Patients were excluded if discharged the same day as ICU admission, if they had ongoing anticoagulant (AC) therapy prior to ICU due to deep venous thrombosis, DVT, and/or PE, or if they had no initial treatment with thromboprophylaxis in the ICU. Patients with chronic
AC therapy at hospital admission, for other reasons than DVT and/or PE, were included in the study.

Data on patients' demography, comorbidities (International classifications of diseases, 10th revision), duration of symptoms, chronic AC therapy, invasive respiratory support, and laboratory values were retrieved from patients' medical records. Data were automatically and manually extracted by medical doctors and all charts and events were validated by at least one additional medical doctor.

\section{Dosing strategies of thromboprophylaxis}

Patients were categorized into three groups according to initial treatment doses of subcutaneous low molecular weight heparin (LMWH) at admission to the ICU. Two different LMWHs, tinzaparin and dalteparin, were used. Tinzaparin and dalteparin are not considered fully interchangeable due to lack of studies establishing equipotent dosing [17]. Dosing strategies were therefore made by classifying the dose according to the recommended dose for the specific indications as recommended by the trade association for the research-based pharmaceutical industry in Sweden in their reference catalogue FASS.se $[18,19]$. Daily doses of tinzaparin and dalteparin were defined as low-dose thromboprophylaxis (2500-4500 international units, IU, tinzaparin or 25005000 IU dalteparin), medium-dose thromboprophylaxis ( $>4500$ IU but $<175$ IU $/ \mathrm{kg}$ of body weight tinzaparin or $>5000 \mathrm{IU}$ but $<200 \mathrm{IU} / \mathrm{kg}$ of body weight dalteparin), and high-dose thromboprophylaxis $(\geq 175 \mathrm{IU} / \mathrm{kg}$ of body weight tinzaparin or $\geq 200 \mathrm{IU} / \mathrm{kg}$ of body weight dalteparin). Patients who received an adjusted dose due to reduced kidney function were classified according to intended dose range. The low dose is the standard thromboprophylaxis used in ICU patients. The medium dose emerged during the pandemic period as the standard thromboprophylaxis dose given twice daily instead of once daily. High dose is the dosage used to treat patients with diagnosed thromboembolic disease [20].

The choice of dosing strategy followed the local recommendations and were modified over time (Additional file 1): In March, low-dose thromboprophylaxis was recommended for all COVID-19 patients at both ICUs. In April, the recommendations were altered to mediumdose and then to high-dose thromboprophylaxis, which was continued throughout the study period in one ICU. In the other ICU, full dose was only used for one week, and then recommendations were altered to medium-dose thromboprophylaxis again. All changes in doses were registered with new dose and date. 


\section{Outcomes}

The primary outcome was 28-day mortality. Days alive and out of ICU at day 28, the cumulative proportion of thromboembolic and bleeding events within 28 days of ICU admission, and maximum levels of Fibrin-D-dimer were used as secondary outcome measures. Thromboembolic events were PE (verified by computed tomography or by clinical suspicion of PE as cause of deterioration combined with findings of acute strain of the right heart on echocardiography), DVT (verified by ultrasound), ischemic stroke (verified by computed tomography), and peripheral arterial embolism (clinical findings of acute peripheral ischemia). Bleeding events were categorized according to the World health organization (WHO) bleeding scale [21-23]: (1) petechiae, tissue hematoma, oropharyngeal bleeding, (2) mild blood loss, hematemesis, macroscopic hematuria, hemoptysis, joint bleeding, (3) gross blood loss requiring red blood cell transfusion and/or hemodynamic instability, (4) debilitating blood loss, severe hemodynamic instability, fatal bleeding, or central nervous system bleeding.

Baseline laboratory values were obtained from $6 \mathrm{~h}$ before to one hour after ICU admission.

\section{Statistical analysis}

Continuous values for baseline and follow-up data are presented in medians with interquartile range (IQR), while categorical or binary data are shown as numbers and proportions. Differences over categories of the exposure were tested with Kruskal-Wallis for continuous data, and Fisher's exact for categorical data. In the survival analyses, participants could accrue follow-up time from date of ICU-admission, to date of death, or when 28 days had passed since admission, whichever occurred first. In analyses of thromboembolic and bleeding events, the date of that event also led to censoring of follow-up time. Kaplan-Meier curves were used to estimate the cumulative risk of death, thromboembolic event, and bleeding event, and the log-rank test was used to compare the initial dosing strategies. Cox proportional hazards regression was used to estimate hazard ratios (HR) with corresponding 95\% confidence intervals (CI) of death within 28 days from ICU admission. Multivariable models were adjusted for sex, age, body mass index (BMI), Simplified Acute Physiology Score III (SAPS III), invasive respiratory support (yes/no), and initial dosing strategy of thromboprophylaxis (low-, medium-, and high-dose thromboprophylaxis) [24,25]. To assess evidence of nonlinearity, the second spline transformation equal to zero was tested as the quantitative covariates were modeled with restricted cubic splines at three knots at fixed percentiles (10th, 50th and 90th) of the distribution of that covariate [26]. As there was no such evidence for age $(p=0.26)$, or SAPS III $(p=0.71)$, those variables were adjusted for in a continuous fashion. Although no formal evidence, there was an indication of nonlinearity between levels of BMI and 28-day mortality $(p=0.08)$, why BMI was categorized as $</ \geq 30 \mathrm{~kg} / \mathrm{m}^{2}$ with a separate category for missing values $(n=6)$. BMI was flexibly adjusted with restricted cubic splines while missing values were accounted for using chained iterations of multiple imputed data sets $(n=20)$ [27]. Although no formal evidence, there was an indication of violation of the assumption of proportional hazards when scaled Schoenfeld residuals were regressed against survival time ( $p=0.06$ for high-dose thromboprophylaxis). Thus, the time-varying effect was fitted by splitting the follow-up time at 7 days from ICU-admission, and fitting the time varying covariate as an interaction term with the main exposure. Statistical significance of interaction was tested using the Wald test. We have conducted sensitivity analyses to investigate the effect of inclusion time, treatment with glucocorticoids and changes in doses of thromboprophylaxis on the result. Two-sided $p<0.05$ was considered statistically significant. Analysis was performed using STATA 13.1 (StataCorp), and R v 3.5.1 (R Core Team (2017). R: A language and environment for statistical computing. R Foundation for Statistical Computing, Vienna, Austria.).

\section{Results}

Out of 165 critically ill COVID-19 patients treated in the ICU due to respiratory failure, 152 remained after exclusion of those with short ICU length of stay $(n=5)$, ongoing AC therapy at ICU admission due to DVT and/ or PE $(n=4)$, or no initial thromboprophylaxis in the ICU $(n=4)$ (Additional file 2). The reason for not giving thromboprophylaxis to four patients was urgent surgery for one patient, but for the other three no reason for not giving thromboprophylaxis could be found in the medical records. Patient characteristics are described in Table 1. Demographic and clinical characteristics did not differ between the three groups. All patients were followed up until death or until the 28th day after ICU-admission. There was no difference between the groups regarding treatment with glucocorticoids: $37.3 \%$ in the lowdose, $45.8 \%$ in medium-dose and $29.7 \%$ in the high-dose groups $(p=0.34)$.

\section{Primary outcome}

For patients who received high-dose prophylaxis, mortality was lower (13.5\%) compared to those who received medium dose $(25.0 \%)$ or low dose $(38.8 \%), p=0.02$, (Table 2). Compared to those who received low-dose thromboprophylaxis, the HR of death was $0.33(95 \%$ CI 0.13-0.87) among those who received high dose, 
and 0.88 (95\% CI 0.43-1.83) among those who received medium dose (Table 3 ). The cumulative proportion of deaths within the first 28 days from ICU admission differed between the three groups ( $p \log$-rank $=0.02)$. The risk of death did not differ between groups of exposure until 7 days after admission, after which the proportion of deaths increased in the low-dose thromboprophylaxis group compared to the other groups (Fig. 1a). When follow-up time was split and the time-varying covariate was fitted as an interaction term with the exposure, the HR of high-dose thromboprophylaxis was 0.08 (95\% CI 0.01-0.62) day 7 to 28 , compared with low-dose thromboprophylaxis the same time period, while no differences were observed during day 0 to 7 (Additional file 3). The interaction term itself was not significant overall $(p=0.18)$. Among the first half of admissions (before April 11, 2020), 74.7\% $(n=59)$ received low-dose and $2.5 \%(n=2)$ high-dose thromboprophylaxis. Corresponding proportions in the second half of admissions was $11.0 \%(n=8)$, and $48 \%(n=35)$ respectively. In analysis with additional adjustment for median admission date, the HR of death was 0.51 (95\% CI 0.14-1.89) of high- vs low-dose thromboprophylaxis, respectively 0.63 (95\% CI 0.24-1.64) comparing the second to the first half of admissions. When adjusting for treatment with systemic glucocorticoids the HR of death was 0.32 (95\% CI $0.12-$ 0.85 ) among those who received high dose, and 0.83 (95\% CI 0.39-1.73) among those who received medium dose, as compared to those who received low-dose thromboprophylaxis (Additional file 4).

\section{Secondary outcomes}

The median number of ICU free days alive during the first 28 days were 0 (IQR 0-22) with low-dose, 11 (IQR 0-19) with medium-dose and 18 (IQR 0-26) with highdose thromboprophylaxis, $(p=0.07)$. The proportion of thromboembolic events was $17.9 \%$ with low dose, $18.8 \%$ with medium dose and $2.7 \%$ with high dose, respectively, $(p=0.04)$. The proportion of bleeding events was $11.9 \%$ with low dose, $14.6 \%$ with medium dose and $2.7 \%$ with high dose, respectively $(p=0.16)$. Cumulative proportions of thromboembolic events, and bleeding events, are depicted in Fig. 1b, c, respectively. In the low-dose thromboprophylaxis group, four patients suffered from ischemic stroke, and two had minor intracranial hemorrhage. There were five major or severe bleeding events, three with low and two with medium-dose thromboprophylaxis (Table 2).

\section{Changes in dose}

Dosing of thromboprophylaxis was registered and followed over time as seen in Additional file 5. Only $5 / 152(3.3 \%)$ of the patients had a reduction of the dose compared to the initial dose. Of the 152 patients 69 (45.4\%) had one or more dose changes during the ICU stay and for 64 patients (42.1\%) the changes included an increase. There were $9(13.0 \%)$ patients who had dose adjustments including both increases and reductions. The median treatment time before a change in dose was 4 (2-7) days. Median duration of thromboprophylaxis was 8 (3-17) days. In the sensitivity analysis excluding patients with a decreased dose compared to initial dose of thromboprophylaxis, the HR was $0.34(0.13-0.90)$ for patients who received high dose as compared with those who received low-dose thromboprophylaxis. When excluding all patients that had any change in dose of thromboprophylaxis the HR was 0.33 (0.11-1.00) for patients who received high dose as compared with those who received low-dose thromboprophylaxis (Additional file 4).

\section{Laboratory results}

While there were no differences in Fibrin-D-dimer, CRP, hemoglobin $(\mathrm{Hb})$, platelet count, and creatinine values at baseline (Table 1), the maximum levels during the first 28 days differed (Table 2). The highest maximum level of Fibrin-D-Dimer was in the group receiving low-dose thromboprophylaxis $(6.4(2.0-14.6))$, the second highest in the group with medium dose (2.8 (1.2-9.4)), and the lowest in the group with high-dose thromboprophylaxis (1.7 (0.7-3.3)), $p=0.002$. The pattern was similar for CRP $(p=0.01)$ and creatinine $(p<0.001)$. For $\mathrm{Hb}(p=0.01)$ and platelet count $(p=0.003)$ the lowest values were found in the group which received low-dose thromboprophylaxis and the highest in the group with high dose. Values over time are presented in Fig. 2.

\section{Discussion}

To the best of our knowledge, this is the first study that has compared three different doses of anticoagulants to critically ill patients with Covid-19 in relation to 28-day mortality, thromboembolism, and bleeding events. The main finding was that those who received high-dose thromboprophylaxis had a $67 \%$ decreased risk of death within the first 28 days compared to those who received low dose. Also, high-dose thromboprophylaxis was associated with a lower incidence of thromboembolic events while no increased risk of bleeding was observed. The 28 -day mortality was $38.8 \%$ with low, $25.0 \%$ with medium and $13.5 \%$ with high-dose thromboprophylaxis which could indicate a dose-response relationship.

The dose of thromboprophylaxis was based on local standardized recommendations and not on degree of critical illness or risk of thrombosis. This is illustrated by the fact that there were no differences in SAPS III score or Fibrin-D-dimer and CRP levels at baseline, and that 
Table 1 Baseline characteristics by initial dosing strategy of thromboprophylaxis

\begin{tabular}{|c|c|c|c|c|c|}
\hline & \multirow[t]{2}{*}{ Total $(n=152)$} & \multicolumn{3}{|c|}{ Initial dosing strategy of thromboprophylaxis } & \multirow[t]{2}{*}{$p$-value ${ }^{d}$} \\
\hline & & Low $\operatorname{dose}^{\mathrm{a}}(n=67)$ & Medium dose ${ }^{b}(n=48)$ & High dose $^{c}(n=37)$ & \\
\hline Age, years & $61(52-69)$ & $63(52-71)$ & $58(51-66)$ & $63(54-70)$ & 0.39 \\
\hline Male sex, No. (\%) & $125(82.2)$ & $59(88.1)$ & $35(72.9)$ & $31(83.8)$ & 0.12 \\
\hline $\mathrm{BMI}, \mathrm{kg} / \mathrm{m}^{2}$ & $28.4(25.8-32.5)$ & $27.7(25.5-30.6)$ & $29.4(26.5-34.3)$ & $28.4(25.1-32.8)$ & 0.10 \\
\hline $\mathrm{BMI} \geq 30$ kg/m², No. (\%) & $58(39.7)$ & $20(31.8)$ & $23(48.9)$ & $15(41.7)$ & 0.18 \\
\hline \multicolumn{6}{|l|}{ Tobacco use } \\
\hline Never, No. (\%) & $79(52.0)$ & $32(47.8)$ & $28(58.3)$ & $19(51.4)$ & 0.53 \\
\hline Former smoker, No. (\%) & $49(32.2)$ & $24(35.8)$ & $12(25.0)$ & $13(35.1)$ & 0.40 \\
\hline Current smoker, No. (\%) & $7(4.6)$ & $3(4.5)$ & $3(6.3)$ & $1(2.7)$ & 0.79 \\
\hline \multicolumn{6}{|l|}{ Comorbidities } \\
\hline Hypertension, №. (\%) & $69(45.4)$ & $32(47.8)$ & $20(41.7)$ & $17(46.0)$ & 0.83 \\
\hline Diabetes without complications, No. (\%) & $19(12.5)$ & $8(11.9)$ & $6(12.5)$ & $5(13.5)$ & 0.99 \\
\hline Diabetes with complications, No. (\%) & $6(4.0)$ & $4(6.0)$ & $0(0.0)$ & $2(5.4)$ & 0.24 \\
\hline Obstructive pulmonary disease, No. (\%) & $30(19.7)$ & $12(17.9)$ & $11(22.9)$ & $7(18.9)$ & 0.81 \\
\hline Ischemic heart disease, No. (\%) & $12(7.9)$ & $6(9.0)$ & $2(4.2)$ & $4(10.8)$ & 0.51 \\
\hline Renal failure, No. (\%) & $9(5.9)$ & $6(9.0)$ & $3(6.3)$ & $0(0.0)$ & 0.17 \\
\hline Liver disease, No. (\%) & $3(2.0)$ & $1(1.5)$ & $1(2.1)$ & $1(2.7)$ & 0.99 \\
\hline Immunosuppression, No. (\%) & $8(5.3)$ & $5(7.5)$ & $1(2.1)$ & $2(5.4)$ & 0.53 \\
\hline Malignancy, №. (\%) & $9(5.9)$ & $6(9.0)$ & $1(2.1)$ & $2(5.4)$ & 0.34 \\
\hline Psychiatric disorder, No. (\%) & $26(17.1)$ & $11(16.4)$ & $9(18.8)$ & $6(16.2)$ & 0.96 \\
\hline \multicolumn{6}{|l|}{ Chronic medication } \\
\hline Vitamin Kantagonist, No. (\%) & $1(0.7)$ & $0(0.0)$ & $0(0.0)$ & $1(2.7)$ & 0.24 \\
\hline Direct oral anticoagulation, №. (\%) & $8(5.3)$ & $3(4.5)$ & $1(2.1)$ & $4(10.8)$ & 0.24 \\
\hline Antiplatelet therapy, №. (\%) & $15(9.9)$ & $9(13.4)$ & $3(6.3)$ & $3(8.1)$ & 0.47 \\
\hline \multicolumn{6}{|l|}{ Duration of symptoms prior to admission } \\
\hline Hospital admission, days & $9(7-12)$ & $9(6-13)$ & $8(6-12)$ & $10(7-14)$ & 0.18 \\
\hline ICU admission, days & $12(8-14)$ & $12(8-14)$ & $10(8-14)$ & $13(10-14)$ & 0.13 \\
\hline SAPS III-score & $56(50-60)$ & $57(53-64)$ & $54(49-59)$ & $55(53-60)$ & 0.25 \\
\hline$<50$, No. (\%) & $34(22.4)$ & $15(22.4)$ & $13(27.1)$ & $6(16.2)$ & 0.53 \\
\hline $50-59$, No. (\%) & $75(44.7)$ & $31(46.3)$ & $25(52.1)$ & $19(51.4)$ & 0.76 \\
\hline$\geq 60$, No. (\%) & $43(28.3)$ & $21(31.3)$ & $10(20.8)$ & $12(32.4)$ & 0.41 \\
\hline Invasive Respiratory support, No. (\%) & $104(68.4)$ & $50(74.6)$ & $34(70.8)$ & $20(54.1)$ & 0.09 \\
\hline \multicolumn{6}{|c|}{ Coagulation, Inflammatory and creatinine tests at baseline } \\
\hline Fibrin-D-dimer, mg/L FEU & $1.5(0.90-3.9)$ & $1.8(1.1-8.4)$ & $1.1(0.7-2.4)$ & $1.3(0.7-2.9)$ & 0.12 \\
\hline $\mathrm{CRP}, \mathrm{mg} / \mathrm{L}$ & $195(134-270)$ & $198(134-268)$ & $203(151-255)$ & $183(130-287)$ & 0.81 \\
\hline Hemoglobin, g/L & $129(118-136)$ & $127(118-137)$ & $130(119-137)$ & $127(113-135)$ & 0.39 \\
\hline Creatinine, $\mu \mathrm{mol} / \mathrm{L}$ & $70(57-88)$ & $74(61-92)$ & $69(48-87)$ & $62(50-74)$ & 0.12 \\
\hline Platelet count, $10^{9} / \mathrm{L}$ & $264(195-339)$ & $255(183-332)$ & $261(219-318)$ & $302(225-391)$ & 0.16 \\
\hline INR & $1.1(1.0-1.1)$ & $1.1(1.0-1.1)$ & $1.1(1.0-1.1)$ & $1.0(1.0-1.2]$ & 0.89 \\
\hline APTt, s & $26(24-29)$ & $25(24-29)$ & $26(23-27)$ & $27(25-30)$ & 0.32 \\
\hline Fibrinogen, $\mathrm{g} / \mathrm{L}$ & $6.7(5.5-8.0)$ & $6.7(5.6-7.7)$ & $7.2(6.4-8.3)$ & $6.7(5.4-7.7)$ & 0.47 \\
\hline
\end{tabular}

Baseline characteristics of 152 patients admitted to the intensive care unit due to COVID-19 at Södersjukhuset, Stockholm, March 6 to April 30, 2020, by initial dosing strategy with tinzaparin/dalteparin as thromboprophylaxis

Values are medians (interquartile range) unless otherwise indicated. APTt, activated partial thromboplastin time; BMI, body mass index; CRP, C-reactive protein; ICU, intensive care unit; OD, once a day; INR, Prothrombin Time International Normalized Ratio; SAPS III, Simplified Acute Physiology Score III

a tinzaparin, 2500-4500 IU OD; or dalteparin, 2500-5000 IU OD

b tinzaparin, $>4500 \mathrm{IU}$ OD to $<175 \mathrm{IU} / \mathrm{kg}$ of body weight OD; or dalteparin, $>5000 \mathrm{IU}$ OD to $<200 \mathrm{IU} / \mathrm{kg}$ of body weight OD

c tinzaparin, $\geq 175 \mathrm{lU} / \mathrm{kg}$ of body weight OD; or dalteparin, $\geq 200 \mathrm{lU} / \mathrm{kg}$ of body weight OD

d $p$-values for differences across exposure categories were obtained using Fisher's exact test for categorical and Kruskal-Wallis test for continuous data 
Table 2 Outcomes by initial dosing strategy of thromboprophylaxis

\begin{tabular}{|c|c|c|c|c|c|}
\hline & \multirow[t]{2}{*}{ Total $(n=152)$} & \multicolumn{3}{|c|}{ Initial dosing strategy of thromboprophylaxis } & \multirow[t]{2}{*}{$p$-value } \\
\hline & & Low dose ${ }^{\mathrm{a}}(n=67)$ & Medium dose ${ }^{b}(n=48)$ & High dose $^{c}(n=37)$ & \\
\hline \multicolumn{6}{|l|}{ Primary outcome } \\
\hline 28-day mortality, No. (\%) & $43(28.3)$ & $26(38.8)$ & $12(25.0)$ & $5(13.5)$ & 0.02 \\
\hline \multicolumn{6}{|l|}{ Secondary outcomes } \\
\hline $\begin{array}{l}\text { ICU-free days alive during } 28 \text { days from } \\
\text { ICU-admission, days }\end{array}$ & $9(0-21)$ & $0(0-20)$ & $11(0-19)$ & $18(0-26)$ & 0.07 \\
\hline Thromboembolic events $<28$ days, No. (\%) & $22(14.5)$ & $12(17.9)$ & $9(18.8)$ & $1(2.7)$ & 0.04 \\
\hline Pulmonary embolism, №. (\%) & $17(11.2)$ & $10(14.9)$ & $6(12.5)$ & $1(2.7)$ & 0.15 \\
\hline Deep vein thrombosis, №. (\%) & $4(2.6)$ & $1(1.5)$ & $3(6.3)$ & $0(0.0)$ & 0.21 \\
\hline Ischemic stroke, No. (\%) & $4(2.7)$ & $4(6.0)$ & $0(0.0)$ & $0(0.0)$ & 0.16 \\
\hline Other thrombotic event, No. (\%) & $3(2.0)$ & $2(3.0)$ & $1(2.1)$ & $0(0.0)$ & 0.79 \\
\hline Time to event, days & $8(6-17)$ & $8(6-20)$ & $8(6-10)$ & $11(11-11)$ & 0.61 \\
\hline Bleeding events < 28 days, No. (\%) & $16(10.5)$ & $8(11.9)$ & $7(14.6)$ & $1(2.7)$ & 0.16 \\
\hline Cerebral parenchymal bleeding, №. (\%) & $2(1.3)$ & $2(3.0)$ & $0(0.0)$ & $0(0.0)$ & 0.50 \\
\hline \multicolumn{6}{|l|}{ WHO bleeding assessment score } \\
\hline Grade I-minor, No. (\%) & $8(5.3)$ & $3(4.5)$ & $4(8.3)$ & $1(2.7)$ & 0.58 \\
\hline Grade II—moderate, No. (\%) & $3(2.0)$ & $2(3.0)$ & $1(2.1)$ & $0(0.0)$ & 0.79 \\
\hline Grade III—major, No. (\%) & $2(1.3)$ & $1(1.5)$ & $1(2.1)$ & $0(0.0)$ & 0.99 \\
\hline Grade IV—severe, No. (\%) & $3(2.0)$ & $2(3.0)$ & $1(2.1)$ & $0(0.0)$ & 0.79 \\
\hline Time to bleeding event, days & $13(8-18)$ & $16(6-20)$ & $11(10-20)$ & $1(1-1)$ & 0.36 \\
\hline \multicolumn{6}{|l|}{ Lab characteristics } \\
\hline Fibrin-D-dimer, mg/L FEU, highest & $3.2(1.2-9.9)$ & $6.4(2.0-14.6)$ & $2.8(1.2-9.4)$ & $1.7(0.7-3.3)$ & 0.002 \\
\hline CRP, mg/L, highest & $282(183-381)$ & $335(200-423)$ & $290(201-385)$ & $229(162-319)$ & 0.01 \\
\hline Hemoglobin, g/L, lowest & $98(85-113)$ & $94(80-104)$ & $102(94-116)$ & $107(95-118)$ & 0.01 \\
\hline Creatinine, $\mu \mathrm{mol} / \mathrm{L}$, highest & $82(64-158)$ & 100 (78-236) & $78(55-139)$ & $66(49-74)$ & $<0.001$ \\
\hline Platelet count, $10^{9} / \mathrm{L}$, lowest & $239(180-322)$ & $203(164-282)$ & $257(197-290)$ & $314(218-370)$ & 0.003 \\
\hline INR, highest & $1.1(1.0-1.2)$ & $1.1(1.0-1.2)$ & $1.1(1.0-1.2)$ & $1.0(1.0-1.1)$ & 0.18 \\
\hline
\end{tabular}

Primary and secondary outcomes during the first 28 days among 152 patients admitted to the intensive care unit due to COVID-19 at Södersjukhuset, Stockholm, March 6 to April 30,2020, by initial dosing strategy with tinzaparin/dalteparin as thromboprophylaxis

Values are medians (interquartile range) unless otherwise indicated. $p$-values for differences across exposure categories were obtained using Fisher's exact test for categorical, and Kruskal Wallis for continuous, data. CRP, C-reactive protein; ICU, intensive care unit; OD, once a day; IQR, interquartile range; WHO, World Health Organization

a Tinzaparin, 2500-4500 IU OD; or dalteparin, 2500-5000 IU OD

b Tinzaparin, $>4500$ IU OD to $<175$ IU/kg of body weight OD; or dalteparin, $>5000$ IU OD to $<200 \mathrm{IU} / \mathrm{kg}$ of body weight OD

c Tinzaparin, $\geq 175 \mathrm{IU} / \mathrm{kg}$ of body weight OD; or dalteparin, $\geq 200 \mathrm{IU} / \mathrm{kg}$ of body weight OD

${ }^{d} p$ values for differences across exposure categories were obtained using Fisher's exact test for categorical and Kruskal-Wallis test for continuous data

patient characteristics were similar between groups. As the local recommendations regarding thromboprophylaxis changed over time $45.4 \%$ of patients had a change in dose with the majority being increases and only 3.3\% having a reduction from initial dose at some point during the ICU-stay. The results were tested in two sensitivity analyses regarding change in dose: when excluding patients with a decreased dose compared to initial dose and excluding all patients that had any change in dose. Both analyses showed that there was still a significantly lower risk of death with high compared to low-dose thromboprophylaxis.
Our results are in congruence with another study where lower mortality was seen when mechanically ventilated COVID-19 patients were treated with higher doses of anticoagulation [15].

In a retrospective study by Trinh et al., still in pre-print, outcomes for 244 COVID-19 patients on invasive respiratory support were investigated with respect to dose of thromboprophylaxis. This study reports a 79\% decreased mortality in therapeutic vs prophylactic anticoagulation, but with a nonsignificantly increased risk of bleeding in the therapeutic group. Another recent study reports 21\% incidence of bleeding among 92 critically ill COVID-19 
Table 3 Risk of death by initial dosing strategy of thromboprophylaxis

\begin{tabular}{|c|c|c|c|c|c|c|}
\hline \multirow{2}{*}{$\begin{array}{l}\text { Initial dosing strategy } \\
\text { of thromboprophylaxis }\end{array}$} & \multirow{2}{*}{$\begin{array}{l}\text { No. } \\
\text { of patients }\end{array}$} & \multirow[t]{2}{*}{ Events/person-days } & \multirow{2}{*}{$\begin{array}{l}\text { IR per } 1.000 \\
\text { person-days } \\
(95 \% \mathrm{Cl})\end{array}$} & \multicolumn{3}{|c|}{$\mathrm{HR}(95 \% \mathrm{Cl})$ of death $\leq 28$ days } \\
\hline & & & & Univariable model & Multivariable model $^{\mathbf{a}}$ & $\begin{array}{l}\text { Multivariable } \\
\text { imputed } \\
\text { model }^{\mathbf{b}}\end{array}$ \\
\hline High dose $e^{c}$ & 37 & $5 / 923$ & $5.4(2.3-13.0)$ & $0.31(0.12-0.82)$ & $0.33(0.13-0.87)$ & $0.30(0.11-0.81)$ \\
\hline Medium dose ${ }^{d}$ & 48 & $12 / 1182$ & $10.2(5.8-17.9)$ & $0.59(0.30-1.16)$ & $0.88(0.43-1.83)$ & $0.87(0.42-1.82)$ \\
\hline Low dose $e^{e}$ & 67 & $26 / 1453$ & $17.9(12.2-26.3)$ & 1.00 (Ref.) & 1.00 (Ref.) & 1.00 (Ref.) \\
\hline
\end{tabular}

Risk of death during the first 28 days among 152 patients admitted to the intensive care unit due to COVID-19 at Södersjukhuset, Stockholm, March 6 to April 30, 2020 , by initial dosing strategy with tinzaparin/dalteparin as thromboprophylaxis

$\mathrm{Cl}$, confidence interval; IR, Incidence Rate; HR, Hazard Ratio

a Adjusted for sex, age (continuously), body mass index $\left(</ \geq 30 \mathrm{~kg} / \mathrm{m}^{2}\right.$ and missing $\left.[n=6]\right)$, invasive mechanical ventilation (yes/no), and Simplified Acute Physiology Score III (continuously)

b Adjusted like the multivariable model but with body mass index imputed due to missing values $(n=6)$, and flexibly modeled with restricted cubic splines at three knots over the percentile $\left(10^{\text {th }}, 50^{\text {th }}\right.$, and $\left.90^{\text {th }}\right)$ distribution of body mass index in the population

c Tinzaparin, $\geq 175 \mathrm{IU} / \mathrm{kg}$ of body weight per daily, or dalteparin, $\geq 200 \mathrm{IU} / \mathrm{kg}$ of body weight daily

d Tinzaparin, $>4500 \mathrm{IU}$ daily to $<175 \mathrm{IU} / \mathrm{kg}$ of body weight daily, or dalteparin, $>5000 \mathrm{IU}$ daily to $<200 \mathrm{IU} / \mathrm{kg}$ of body weight daily

e Tinzaparin, 2500-4500 IU daily, or dalteparin, 2500-5000 IU daily

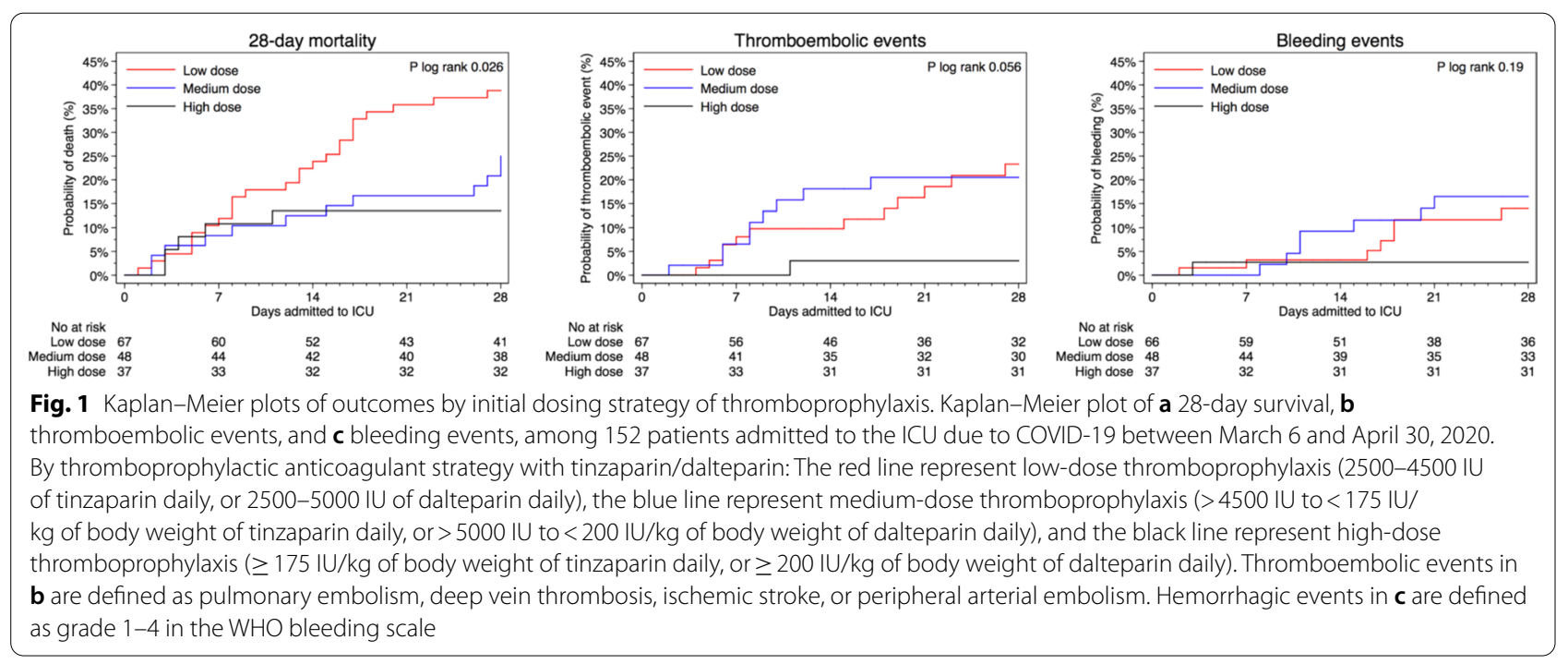

patients, of which 43 had prophylactic dose and 49 had full-dose anticoagulation [16].

This is contrary to our findings of lower proportions of bleeding events in the high-dose group. This difference between the groups was not statistically significant, but still raises questions about possible pathophysiological mechanisms. The unusually severe hypercoagulable state in COVID-19 has been suggested to be caused by bidirectional cross-talk between inflammation and thrombosis; direct viral endothelial activation in the lungs, release of inflammatory cytokines that activate expression of tissue factor with platetet activation and thrombin generation [28]. Perhaps the treatment with high-dose LMWH can reduce both inflammation and coagulation.
If the coagulation is less triggered, there will be less fibrin deposits that activate the fibrinolysis, leading to less bleeding.

Subclinical bleeding could potentially impact the safety of high-dose thromboprophylaxis. Hemoglobin values are known to drop during intensive care. As many as $97 \%$ of patients are anemic after a week in ICU care $[29,30]$. Decreased erythropoiesis, frequent blood sampling and bleeding are known reasons why anemia is common among ICU patients [31]. Interestingly, no signs were found of more severe anemia in patients with higher doses of anticoagulation, perhaps because COVID-19 patients are hypercoagulable and might not bleed easily despite high-dose thromboprophylaxis. 

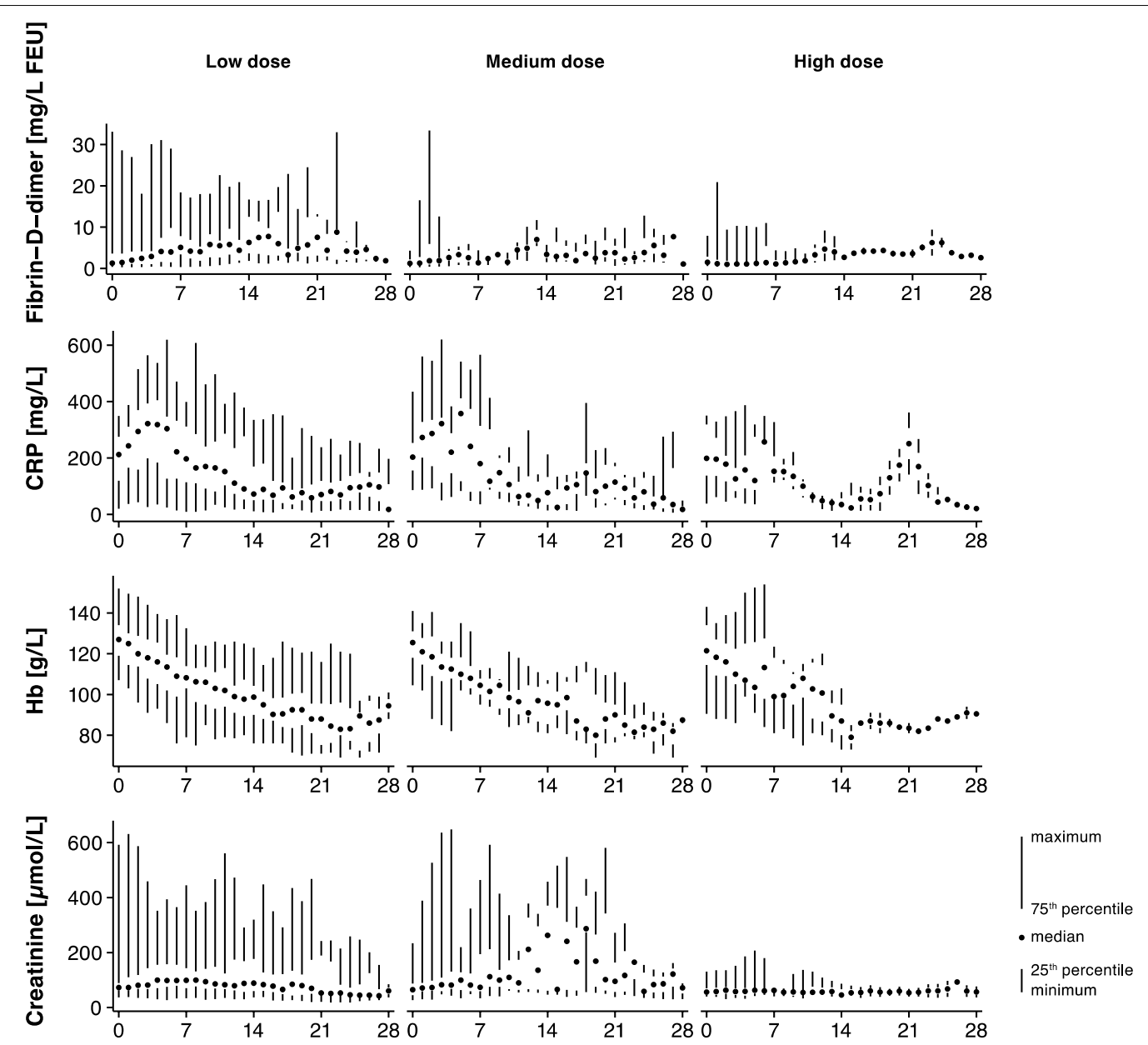

Day from ICU admission

Fig. 2 Laboratory markers by initial dosing strategy of thromboprophylaxis. Laboratory markers Fibrin-D-Dimer, C-reactive protein (CRP), hemoglobin concentration, and creatinine as a function of day from admission are shown as median, interquartile range and range. Columns are low-dose thromboprophylaxis (2500-4500 IU of tinzaparin daily, or 2500-5000 IU of dalteparin daily), medium-dose thromboprophylaxis (> 4500 IU to $<175 \mathrm{IU} / \mathrm{kg}$ of body weight of tinzaparin daily, or $>5000 \mathrm{IU}$ to $<200 \mathrm{IU} / \mathrm{kg}$ of body weight of dalteparin daily), and high-dose thromboprophylaxis ( $\geq 175 \mathrm{IU} / \mathrm{kg}$ of body weight of tinzaparin daily, or $\geq 200 \mathrm{IU} / \mathrm{kg}$ of body weight of dalteparin daily)

A large multi-center trial showed a reduced 28-day mortality when treating patients on mechanical ventilation and supplementary oxygen with dexamethasone [32]. In our cohort $58(38.2 \%)$ patients had at least one dose of systemic corticosteroids. At the time of our study corticosteroids were not yet recommended for treating the inflammation caused by COVID-19. Instead, corticosteroids were given to patients for other reasons, e.g. to reduce risk of adrenal crisis in patients on chronic therapy [33] and to counteract vasoplegia in patients with septic shock [34]. The proportion of patients with systemic corticosteroids did not differ between groups with low, medium, or high-dose thromboprophylaxis $(p=0.34)$ and adjusting for corticosteroids did not alter the result.

\section{Strengths and limitations}

The present study lacks the rigor of a prospective randomized design. The study groups thus reflect the progression from low to high-dose thromboprophylaxis based on modifications of local clinical guidelines over time. As the study is observational, other factors might have affected outcome. During the study period, the ventilation strategy was changed from a classical acute respiratory distress syndrome strategy with low tidal volumes, fluid restriction, and heavy sedation to a more liberal strategy allowing higher tidal volumes, more 
fluids and less sedation. However, in analysis with additional adjustment for median admission date, the risk of death was still $49 \%$ lower (although not statistically significant) among those who received high dose compared with low-dose prophylaxis. Furthermore, patients were grouped according to initial dose of thromboprophylaxis at admission to the ICU, and outcomes in relation to total dose thromboprophylaxis received have not been analyzed. However, the results were robust when excluding patients who had a decrease in dose compared to initial dose and when excluding patients who had any change in dose. There was also a lower proportion of patients on invasive ventilation in the group with high dose, though not statistically significant, and this was adjusted for in the statistical analysis.

Due to the heavy work load during the pandemic, risk of complications during intrahospital transportation of critically ill patients due to respiratory instability, and the risk of viral contamination of radiology suites, it was early on not always possible to perform computed tomography-scans to diagnose PE or Pulmonary Thromboses, PT. As the number of PE/PT might be underestimated, decision was made to use mortality as primary outcome.

The results regarding risk of bleeding should be interpreted with caution as the sample size is too small to estimate rare complications. Thus, if high-dose thromboprophylaxis is used, it is important to continue to monitor the patients closely for potential bleeding.

\section{Conclusions}

Among critically ill COVID-19 patients with respiratory failure, high-dose thromboprophylaxis was associated with a lower risk of death and a lower cumulative incidence of thromboembolic events compared with lower doses. This study suggests that high-dose thromboprophylaxis should be tested in a randomized trial in critically ill COVID-19 patients.

\section{Supplementary information}

Supplementary information accompanies this paper at https://doi. org/10.1186/s13054-020-03375-7.

\section{Additional file 1. Local guidelines. \\ Additional file 2. Flow chart. \\ Additional file 3. Time varying effect. \\ Additional file 4. Sensitivity analyses}

Additional file 5. Individual does of thromboprophylaxis over time.

\section{Abbreviations}

COVID: Coronavirus disease; ICU: Intensive care units; CRP: C-reactive peptide; SARS-CoV-2: Severe acute respiratory distress syndrome Coronavirus 2; AC: Anticoagulant; DVT: Deep venous thrombosis; PE: Pulmonary embolism; LMWH: Low molecular weight heparin; IU: International units; WHO: World health organization; IQR: Interquartile range; HR: Hazard ratio; Cl: Confidence intervals; BMI: Body mass index; SAPS: Simplified acute physiology score; $\mathrm{Hb}$ : Hemoglobin; kg: Kilogram; APTt: Activated partial thromboplastin time; INR: Prothrombin time international normalized ratio; IR: Incidence Rate; FEU: Fibrinogen equipotent unit; PT: Pulmonary thromboses.

\section{Acknowledgements}

We would like to thank W. Müller for his whole-hearted support in data collection from electronic sources.

\section{Authors' contributions}

Concept was done by S.J., J.H., M.D., O.S., J.L., Å.H.E., A.S., M.C. Data collection was performed by S.J., J.H., M.D., O.S., J.L., A..H.E., A.S., M.G., M.C. Data analysis was done by O.S., H.J.P. S.J., J.H., M.D., O.S., J.L., Å.H.E., A.S., M.S., H.J.P., M.C interpreted the data. Drafting of the manuscript was done by S.J., M.C. Editing of the manuscript was done by S.J., J.H., M.D., O.S., J.L., Å.H.E., A.S., M.S., H.J.P., M.G., M.C. M.C., S.J., O.S. had full access to all the data in the study and take responsibility for the integrity of the data and the accuracy of the data analysis. All authors read and approved the final manuscript.

Funding

Open access funding provided by Karolinska Institute.

\section{Availability of data and materials}

The datasets generated and analyzed during current study are not publicly available due to patient records' regulations but can be made available from corresponding author on request.

\section{Ethics approval and consent to participate}

The study was approved by the regional ethical review board in Uppsala, Sweden, (Dnr: 2020-01302, amendment 2020-02890) and informed consent was waived

\section{Consent for publication}

Not applicable.

\section{Competing interests}

M. Cronhjort has honoraria for lectures from B. Braun. With this exception the authors declare that they have no competing interests.

\section{Author details}

${ }^{1}$ Department of Clinical Science and Education, Södersjukhuset, Karolinska Institutet, Stockholm, Sweden. ${ }^{2}$ Department of Anaesthesia and Intensive Care, Södersjukhuset, Stockholm, Sweden. ${ }^{3}$ Department of Medicine, Center for Resuscitation Science, Karolinska Institutet, Stockholm, Sweden. ${ }^{4}$ Department of Surgery, Södersjukhuset, Stockholm, Sweden. ${ }^{5}$ Institute of Environmental Medicine, Unit of Cardiovascular and Nutritional Epidemiology, Karolinska Institutet, Stockholm, Sweden. ${ }^{6}$ Department of Internal Medicine, Södersjukhuset, Stockholm, Sweden. ${ }^{7}$ Department of Perioperative Medicine and Intensive Care, Karolinska University Hospital, Stockholm, Sweden.

${ }^{8}$ Department of Clinical Science, Intervention and Technology (CLINTEC), Karolinska Institutet, Stockholm, Sweden. ${ }^{9}$ Department of Molecular Medicine and Surgery, Karolinska Institutet, Stockholm, Sweden.

Received: 1 September 2020 Accepted: 4 November 2020 Published online: 23 November 2020

References

1. Joly BS, Siguret V, Veyradier A. Understanding pathophysiology of hemostasis disorders in critically ill patients with COVID-19. Intensive Care Med. 2020;46:1603-6.

2. Lodigiani C, lapichino G, Carenzo L, Cecconi M, Ferrazzi P, Sebastian T, et al. Venous and arterial thromboembolic complications in COVID-19 patients admitted to an academic hospital in Milan, Italy. Thromb Res. 2020;191:9-14

3. Wichmann D, Sperhake JP, Lutgehetmann M, Steurer S, Edler C, Heinemann $A$, et al. Autopsy findings and venous thromboembolism in patients with COVID-19. Ann Intern Med. 2020;173(4):268-77. 
4. Varga Z, Flammer AJ, Steiger P, Haberecker M, Andermatt R, Zinkernagel AS, et al. Endothelial cell infection and endotheliitis in COVID-19. Lancet 2020;395(10234):1417-8.

5. Ackermann M, Verleden SE, Kuehnel M, Haverich A, Welte T, Laenger F, et al Pulmonary vascular endothelialitis, thrombosis, and angiogenesis in Covid19. N Engl J Med. 2020;383(2):120-8.

6. Klok FA, Kruip M, van der Meer NJM, Arbous MS, Gommers D, Kant KM et al. Incidence of thrombotic complications in critically ill ICU patients with COVID-19. Thromb Res. 2020a;191:145-7.

7. Klok FA, Kruip M, van der Meer NJM, Arbous MS, Gommers D, Kant KM, et al. Confirmation of the high cumulative incidence of thrombotic complications in critically ill ICU patients with COVID-19: an updated analysis. Thromb Res. 2020b;191:148-50.

8. Helms J, Tacquard C, Severac F, Leonard-Lorant I, Ohana M, Delabranche $X$, et al. High risk of thrombosis in patients with severe SARS-CoV-2 infection: a multicenter prospective cohort study. Intensive Care Med. 2020;46(6):1089-98.

9. Llitjos JF, Leclerc M, Chochois C, Monsallier JM, Ramakers M, Auvray M, et al. High incidence of venous thromboembolic events in anticoagulated severe COVID-19 patients. J Thromb Haemost. 2020;18:1743-6.

10. Levi M, Thachil J, Iba T, Levy JH. Coagulation abnormalities and thrombosis in patients with COVID-19. Lancet Haematol. 2020;7(6):e438-40.

11. Thachil J, Tang N, Gando S, Falanga A, Cattaneo M, Levi M, et al. ISTH interim guidance on recognition and management of coagulopathy in COVID-19. J Thromb Haemost. 2020;18(5):1023-6.

12. Tang N, Li D, Wang X, Sun Z. Abnormal coagulation parameters are associated with poor prognosis in patients with novel coronavirus pneumonia. J Thromb Haemost. 2020;18(4):844-7.

13. Fogarty H, Townsend L, Ni Cheallaigh C, Bergin C, Martin-Loeches I, Browne $\mathrm{P}$, et al. COVID19 coagulopathy in Caucasian patients. Br J Haematol. 2020;189(6):1044-9.

14. Tang N, Bai H, Chen X, Gong J, Li D, Sun Z. Anticoagulant treatment is associated with decreased mortality in severe coronavirus disease 2019 patients with coagulopathy. J Thromb Haemost. 2020;18(5):1094-9.

15. Paranjpe I, Fuster V, Lala A, Russak A, Glicksberg BS, Levin MA, et al. Association of treatment dose anticoagulation with in-hospital survival among hospitalized patients with COVID-19. J Am Coll Cardiol. 2020;76:122-4.

16. Fraisse M, Logre E, Pajot $\mathrm{O}$, Mentec $\mathrm{H}$, Plantefeve G, Contou D. Thrombotic and hemorrhagic events in critically ill COVID-19 patients: a French monocenter retrospective study. Crit Care. 2020;24(1):275.

17. Amin A. Therapeutic interchange of parenteral anticoagulants: challenges for pharmacy and therapeutics committees. Pharmaceuticals (Basel). 2011;4(11):1475-87.

18. FASS. Innohep. https://www.fass.se/LIF/product?userType $=0 \&$ nplld $=19910$ 8090000102020

19. FASS. Fragmin. https://www.fass.se/LIF/product?userType $=0 \& n$ plld $=19880$ 3180000402020
20. Lee AYY, Kamphuisen PW, Meyer G, Bauersachs R, Janas MS, Jarner MF, et al. Tinzaparin vs warfarin for treatment of acute venous thromboembolism in patients with active cancer: a randomized clinical trial. JAMA. 2015;314(7):677-86.

21. Fogarty PF, Tarantino MD, Brainsky A, Signorovitch J, Grotzinger KM. Selective validation of the WHO Bleeding Scale in patients with chronic immune thrombocytopenia. Curr Med Res Opin. 2012;28(1):79-87.

22. Miller $A B$, Hoogstraten $B$, Staquet $M$, Winkler $A$. Reporting results of cancer treatment. Cancer. 1981:47(1):207-14.

23. Slichter SJ, Kaufman RM, Assmann SF, McCullough J, Triulzi DJ, Strauss RG, et al. Dose of prophylactic platelet transfusions and prevention of hemorrhage. N Engl J Med. 2010;362(7):600-13.

24. Metnitz PG, Moreno RP, Almeida E, Jordan B, Bauer P, Campos RA, et al. SAPS 3 - from evaluation of the patient to evaluation of the intensive care unit. Part 1: objectives, methods and cohort description. Intensive Care Med. 2005;31(10):1336-44.

25. Moreno RP, Metnitz PG, Almeida E, Jordan B, Bauer P, Campos RA, et al. SAPS 3 - from evaluation of the patient to evaluation of the intensive care unit. Part 2: development of a prognostic model for hospital mortality at ICU admission. Intensive Care Med. 2005;31(10):1345-55.

26. Orsini NGS. A procedure to tabulate and plot results after flexible modeling of a quantitative covariates. Stata J. 2011;11(1):1-29.

27. White IR, Royston P, Wood AM. Multiple imputation using chained equations: issues and guidance for practice. Stat Med. 2011;30(4):377-99.

28. Abou-Ismail MY, Diamond A, Kapoor S, Arafah Y, Nayak L. The hypercoagulable state in COVID-19: incidence, pathophysiology, and management. Thromb Res. 2020;194:101-15.

29. Thomas J, Jensen L, Nahirniak S, Gibney RT. Anemia and blood transfusion practices in the critically ill: a prospective cohort review. Heart Lung. 2010;39(3):217-25.

30. Hajjar LA, Auler Junior JO, Santos L, Galas F. Blood tranfusion in critically ill patients: state of the art. Clinics (Sao Paulo). 2007;62(4):507-24.

31. Rawal G, Kumar R, Yadav S, Singh A. Anemia in intensive care: a review of current concepts. J Crit Care Med (Targu Mures). 2016;2(3):109-14.

32. Group RC, Horby P, Lim WS, Emberson JR, Mafham M, Bell JL, et al. Dexamethasone in hospitalized patients with Covid-19-preliminary report. N Engl J Med. 2020. https://doi.org/10.1056/NEJMoa2021436.

33. Calissendorff J, Blomqvist H. Secondary cortisol insufficiency —an undiagnosed condition. Lakartidningen. 2007;104(41):2965-8.

34. Venkatesh B, Finfer S, Cohen J, Rajbhandari D, Arabi Y, Bellomo R, et al. Adjunctive glucocorticoid therapy in patients with septic shock. N Engl J Med. 2018;378(9):797-808.

\section{Publisher's Note}

Springer Nature remains neutral with regard to jurisdictional claims in published maps and institutional affiliations.
Ready to submit your research? Choose BMC and benefit from:

- fast, convenient online submission

- thorough peer review by experienced researchers in your field

- rapid publication on acceptance

- support for research data, including large and complex data types

- gold Open Access which fosters wider collaboration and increased citations

- maximum visibility for your research: over $100 \mathrm{M}$ website views per year

At BMC, research is always in progress.

Learn more biomedcentral.com/submissions 